\title{
Feasibility of eradication of mutans streptococci from oral cavities
}

\author{
Yoshiaki Nomura ${ }^{\S, \dagger}$, Hiroaki Takeuchi ${ }^{\S}$, Noboru Kaneko ${ }^{\ddagger}$, Khairul Matin ${ }^{\S}$, \\ Ritsuko Iguchi ${ }^{\text {II }}$, Yoshihiro Toyoshima ${ }^{\star}$, Yoshiharu Kono ${ }^{\text {II }}$, Takuji Ikemi ${ }^{\text {II }}$, \\ Susumu Imai ${ }^{\S}$, Toshiki Nishizawa ${ }^{\S}$, Kazuo Fukushima\# \\ and Nobuhiro Hanada ${ }^{\S}$

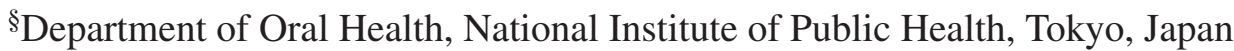 \\ tDepartment of Preventive Dentistry and Public Health, \\ Tsurumi University School of Dental Medicine, Yokohama, Japan

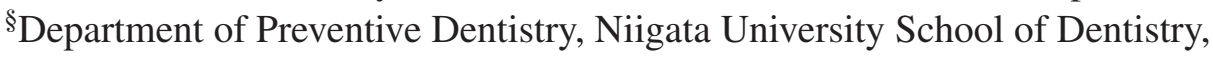 \\ Niigata, Japan \\ Departments of ${ }^{\mathbb{I}}$ Operative Dentistry and ${ }^{\#}$ Microbiology, \\ Nihon University School of Dentistry at Matsudo, Chiba, Japan \\ *Dai-ichi Mutual Life Insurance Company, Hibiya Medical Center, Tokyo, Japan
}

(Received 15 April and accepted 29 July 2004)

\begin{abstract}
Objectives: Dental caries prevention programs using chlorhexidine (CHX) have been proposed, but CHX's effect in reducing levels of mutans streptococci ( $S$. mutans and $S$. sobrinus) appears to last for only a few months. The aim of this study was to attempt to eradicate mutans streptococci from the oral cavity using intensive professional mechanical tooth cleaning (PMTC) and topical application of CHX in custom-made trays. Methods: Seven adult dentate subjects participated in this study (mean age $53.7+/$ 5.6, age range 46 to 62, mean DMFT, 9.1 +/- 4.2). For each subject, PMTC was carried out eight times within ten days. After each PMTC, 1\% CHX was applied twice to the tooth surface using custom-made trays. In addition, as home treatment, subjects were required to carry out tooth brushing three times a day, and apply $0.2 \% \mathrm{CHX}$ in custom trays after brushing in the morning and evening. In addition, subjects rinsed with 0.2\% CHX solution after lunch. Salivary levels of mutans streptococci were evaluated using Dentocult-
\end{abstract}

Correspondence to Dr. Yoshiaki Nomura, Department of Preventive Dentistry and Public Health, Tsurumi University, 21-3, Tsurumi, Tsurumi-ku, Yokohama, Kanagawa 230-8501, Japan Tel: +81-45-580-8379

Fax: +81-45-573-9599

E-mail: nomura-y@tsurumi-u.ac.jp
SM at baseline and on days 9, 20, 70, 120. Results: Mutans streptococci were eradicated by day 120 from 4 of the 7 seven subjects participating in this study. Those 3 subjects still harboring mutans streptococci exhibited deep periodontal pocketing. Conclusions: Eradication of mutans streptococci from the oral cavity is feasible using a combination of $\mathrm{CHX}$ application in custom-made trays and intensive PMTC. (J. Oral Sci. 46, 179-183, 2004)

Keywords: mutans streptococci; chlorhexidine; eradication.

\section{Introduction}

It is generally accepted that harboring mutans streptococci (Streptococcus mutans and Streptococcus sobrinus) is a risk factor for dental caries. A number of in-vitro studies have demonstrated mechanisms by which these bacteria play a role in dental caries formation (1). In this regard, the production of water-insoluble glucans on the tooth surface seems a particularly important virulence factor (2-4).

Dental plaque is a biofilm, and the surface of it is covered with the matrix structure of dextran (5-8), which is resistant to penetration by most anti-microbial agents. 
Chlorhexidine (CHX) is a topical anti-microbial agent that is effective in the elimination of mutans streptococci from the oral cavity (9). Application of CHX is performed using a rinsing solution, dentifrice, varnish on the tooth surface or gel application in individual trays. Of these methods, varnish application to the tooth surface and gel application using individual trays have been shown to be most effective $(10,11)$ since they permit a high concentration of CHX to be maintained on the tooth surface. However, even using these methods, this agent is still unable to penetrate the biofilm (11). Eradication of mutans streptococci using $\mathrm{CHX}$ has been attempted in the clinical trial setting, but re-growth of the bacteria is generally observed within two to three months (12). There are no reports of permanent eradication of mutans streptococci from the oral cavity. Moreover, attempting to eradiate these bacteria using high concentrations of CHX over an extended time frame may result in local adverse effects on the oral mucosa $(13,14)$.

We propose use of a combination of professional mechanical tooth cleaning (PMTC) and a dental drug delivery system (3DS) with CHX for elimination of mutans streptococci from the oral cavity $(15,16)$. 3DS consists of drug retainers that contact the tooth surface and permit contact of the anti-microbial drugs directly with the tooth surface. Since mutans streptococci do not have receptors to permit adherence to the oral mucosa (17), it is not necessary to eradicate them from areas where teeth are absent. It was hypothesized PMTC would destroy the biofilm structure and permit the delivered $\mathrm{CHX}$ to reach the mutans streptococci remaining in micro-colonies.

\section{Materials and Methods}

\section{Subjects and clinical examination}

This study was approved by the ethical committee of the National Institute of Infectious Diseases of Japan. Members of the institute and co-researchers of this study were recruited, and all subjects gave written informed consent for participation in the study. In total, seven adult male subjects participated, with mean age $53.7 \pm 5.6$ years and mean DMFT 9.1 \pm 4 .2. Probing depths were measured a six sites per tooth using a WHO probe. Among the seven subjects, 2 subjects had deep periodontal pockets ( $>4$ $\mathrm{mm}$ ). All subjects exhibited pockets of $3 \mathrm{~mm}$.

\section{Clinical and sampling procedures}

Levels of salivary mutans streptococci were determined at baseline using Dentocult-SM strip methods (Orion Diagnostica, Finland). Alginate impressions were then taken and maxillary and mandible casts prepared. A polypropylene sheet $(3.0 \mathrm{~mm}$ disk for mouth guard soft,
Keystone, New Jersey, U.S.A.) was vacuum-adapted to each cast with a vacuum-forming machine (VACCUM ADAPTER I, Keystone). Vacuum-adapted drug retainers were individually fabricated to cover the complete arch of the dentition. The drug retainer was trimmed to be approximately $1.0 \mathrm{~mm}$ apical to the gingival margin.

Before CHX application, PMTC was carried out eight times within 10 days on each subject to remove the tooth biofilm. By the PMTC, dental plaque was disclosed prior to its removal using rubber cups with polishing paste (Prophy Paste;(RDA170); CCS Cleanchemical, Vasby, Sweden). The remaining inter-dental plaque was removed using dental floss and polishing using Eva chips with polishing paste. Complete plaque removal was confirmed by further disclosing. The tooth surface was varnished with a $0.2 \% \mathrm{NaF}$ solution (FULORIDENT GEL, Stone Pharmaceuticals, Philadelphia, USA).

After each PMTC, 1\% CHX gel (CORSODYL Zahngel, Smithkline Beecham, Thorishaus, Switzerland) was injected into those periodontal pockets deeper than $4 \mathrm{~mm}$. This gel was also applied using a dental drug retainer for 5 minutes. During the 10 days after PMTC, subjects also applied 0.2\% CHX gel (Plakout, Howe-Neos Dental, Bioggio, Switzerland) twice a day after tooth brushing (morning and evening) using a custom-made tray. In addition, after lunch, tooth brushing and mouth rinsing using $0.2 \%$ CHX mouth rinse was performed. Salivary mutans streptococci levels were determined using the DentocultSM system at days 9, 20, 70, 120. On days 70 and 120, bacteria were also cultured to determine salivary mutans streptococci levels.

\section{Microbial procedures and saliva sampling}

Salivary mutans streptococci and Lactobacillus were counted using a commercially available mutans streptococci evaluation kit, Dentocult-SM (Orion Diagnostica, Epsom, Finland). The levels were classified according to the manufacturer's instructions, that is: level $0-1:<100,000$ colony forming units (CFU) mutans streptococci $/ \mathrm{ml}$ saliva; level 2: 100,000 < CFU/ml < 1,000,000; and level 3: > 1,000,000 CFU/ml.

On days 70 and 120, mutans streptococci were also cultured. Paraffin-stimulated whole saliva samples were collected for 5 minutes. Saliva samples of $50 \mu \mathrm{l}$ were then sonicated by ultrasonic dispersion (60 power output) for 10 seconds and spread onto Mitis-Salivarius agar (MS, Gibco, Tokyo, Japan) plates for growth of streptococci, and onto improved Mitis-Salivarius agar plates containing $0.02 \mathrm{M}$ bacitracin (Wako Pure Chemicals, Osaka, Japan) (MSB) for selective growth of mutans streptococci (18), using an EDDY JET spiral system (Gunze Sangyo, Tokyo, 

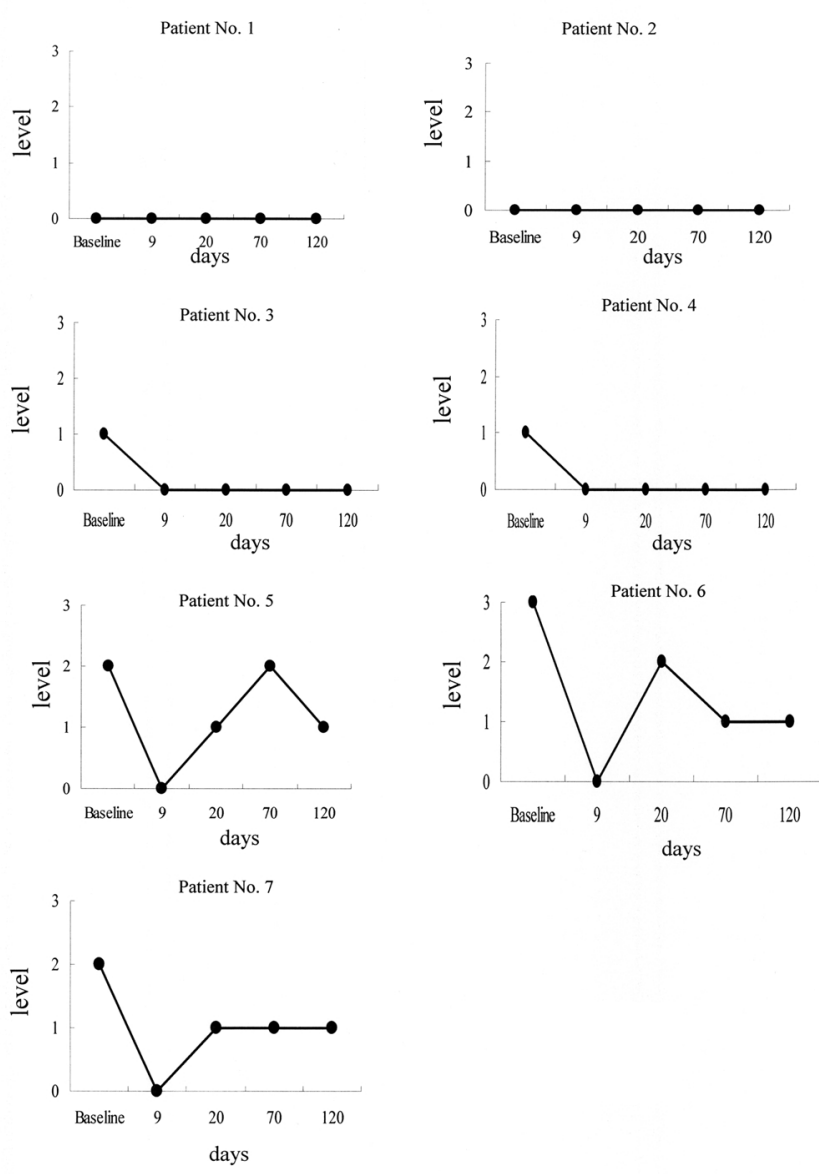

Fig. 1 Evaluation of the salivary levels of mutans streptococci by Dentocult SM.

Japan). After 48 hours anaerobic incubation, colonies were counted and the number of bacteria per $\mathrm{ml}$ of whole saliva calculated.

\section{Statistical analysis}

Mann-Whitney's $U$-tests were used for the evaluation of baseline differences between subjects in whom mutans streptococci was eradiated and those still harboring mutans streptococci.

\section{Results}

Figure 1 shows the mutans streptococci levels evaluated using the Dentocult SM. Two subjects with level 0 (no mutans streptococci detected )at baseline also had level 0 throughout the study. In addition, two subjects with level 1 at baseline decreased to the 0 level after 120 days. Three subjects above level 2 at baseline decreased to level 0 by day 9 , and recovered to level 2 by day 120 .

Figure 2 shows the mutans streptococci levels evaluated by the improved MSB culture system at 70 (A) and 120 days (B). Eradication of mutans streptococci was observed
(A)

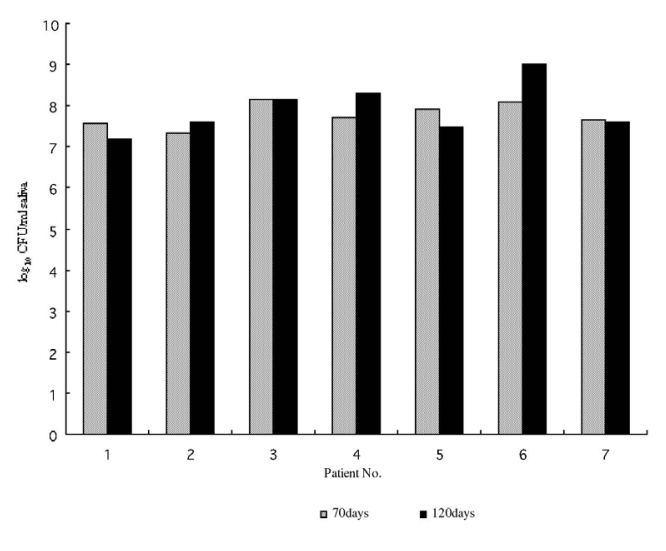

(B)

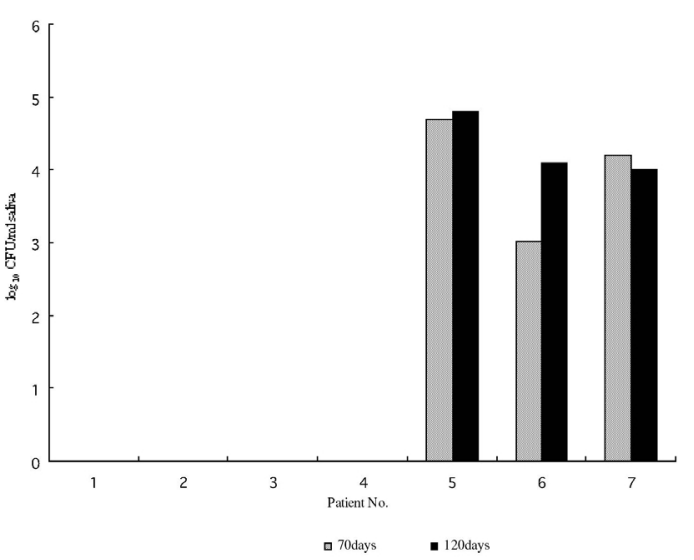

Fig. 2 Confirmation of the eradication of mutans streptococci from the oral cavity by culture.

Table 1 Baseline clinical characteristics (mean $\pm \mathrm{SD}$ ) of subjects according to success of mutans streptococci eradication methods

\begin{tabular}{lccc}
\hline & Eradicated & Not eradicated & \\
& $(\mathbf{n}=\mathbf{4})$ & $(\mathbf{n}=\mathbf{3})$ & \\
\hline DMFT & $10.00 \pm 4.97$ & $8.00 \pm 5.29$ & 0.31 \\
& & & \\
Probing depth (\% of pockets) & & 0.78 \\
$>3 \mathrm{~mm}$ & $49.85 \pm 19.92$ & $54.76 \pm 45.07$ & 0.70 \\
$>4 \mathrm{~mm}$ & $6.40 \pm 2.40$ & $18.85 \pm 18.46$ & 0.71 \\
$>5 \mathrm{~mm}$ & $1.64 \pm 0.89$ & $6.94 \pm 9.57$ & 0.58 \\
\hline $6 \mathrm{~mm}$ & $0.89 \pm 1.03$ & $2.18 \pm 3.78$ & \\
\hline
\end{tabular}

in three subjects.

The baseline characteristics of subjects in whom mutans streptococci were eradicated and those exhibiting mutans streptococci re-growth are shown in Table 1. There was a 
tendency for those subjects with deep periodontal pockets to exhibit streptococci regrowth, although no statistically significant difference was observed.

\section{Discussion}

The results of the present study demonstrate that 3DS used in combination with intensive PMTC is effective in reducing salivary levels of mutans streptococci and in eradicating mutans streptococci. A number of dental caries preventive programs have been described (for review see Lewis et al.;19), including tooth cleaning, and fluoride or CHX application. Tooth cleaning and tooth brushing instruction have some benefit in caries prevention, although this benefit appears minor (20). A combination of oral hygiene instruction and fluoride application appears more effective (21), and if CHX is used in addition, an even greater favorable effect is evident (11). However, these preventive measures do not appear to result in complete inhibition of new dental caries.

Complete removal of dental plaque can be challenging, even in the case of chemical plaque removal (22). Tooth brushing in combination with tooth brushing instruction is also of questionable value, especially on tooth surfaces prone to caries (23). PMTC has been suggested as being one of the most effective methods for plaque removal, and PMTC performed by dental hygienists has been demonstrated to suppress dental caries $(24,25)$ and reduce salivary levels of the mutans streptococci without the use of anti-microbial agents (26).

Elimination of mutans streptococci using $\mathrm{CHX}$ has been attempted in a number of studies, but the mean levels of these bacteria returned to baseline levels within two weeks when a rinsing solution was used (27), within 4 weeks using gel application or within 12 weeks using varnish (28). The results of these studies suggest that eradication of mutans streptococci from the oral cavity is not feasible. However, these results might be a consequence of inadequate plaque removal prior to $\mathrm{CHX}$ application. The dental plaque biofilm in which mutans streptococci are found (8) is resistant to anti-microbial agent penetration (29). Using the system described in the current study, pre-treatment of the tooth surface using PMTC to remove the biofilm appears to permit CHX to function more effectively in mutans streptococci removal.

The use of either PMTC or anti-microbial agents alone has some benefit in preventing dental caries, but is not effective in eradication of mutans streptococci. Using the system described in this study, that is complete removal of dental plaque by intensive PMTC and frequent applications of CHX within a short period of time, in some cases results in the eradication of mutans streptococci from the oral cavity. However, even using this system, mutans streptococci were eradicated from only 4 of 7 subjects.

Baseline differences in the periodontal condition between subjects in whom mutans streptococci were eradicated and those in whom they were not eradicated were not seen. This may be the result of the small sample size, and thus insufficient power to detect differences. However, in general, those subjects where mutans streptococci were not eradicated had deeper periodontal pockets. These results suggest periodontal treatment might be advisable prior to use of anti-microbial agents.

In conclusion, using appropriate methods, eradication of mutans streptococci from the oral cavity is feasible, but is not consistently achieved.

\section{References}

1. Kuramitsu HK (1987) Recent advances in defining the cariogenicity of mutans streptococci: molecular genetic approaches. Eur J Epidemiol 3, 257-260

2. Inoue M, Smith EE (1980) Specific inhibition of glucosyltransferase of Streptococcus mutans. Carbohydr Res 80, 163-177

3. Montville TJ, Cooney CL, Sinskey AJ (1977) Measurement and synthesis of insoluble and soluble dextran by Streptococcus mutans. J Dent Res 56, 983-989

4. Takada K, Shiota T, Curtiss R, Michalek SM (1985) Inhibition of plaque and caries formation by a glucan produced by Streptococcus mutans mutant UAB108. Infect Immun 50, 833-843

5. Shu M, Wong L, Miller JH, Sissons CH (2000) Development of multi-species consortia biofilms of oral bacteria as an enamel and root caries model system. Arch Oral Biol 45, 27-40

6. Sissons CH, Wong L, Shu M (1998) Factors affecting the resting $\mathrm{pH}$ of in vitro human microcosm dental plaque and Streptococcus mutans biofilms. Arch Oral Biol 43, 93-102

7. Rose RK, Turner SJ (1998) Extracellular volume in streptococcal model biofilms: effects of $\mathrm{pH}$, calcium and fluoride. Biochim Biophys Acta 1379, 185190

8. Marsh PD, Bradshaw DJ (1997) Physiological approaches to the control of oral biofilms. Adv Dent Res 11, 176-185

9. Emilson CG (1994) Potential efficacy of chlorhexidine against mutans streptococci and human dental caries. J Dent Res 73, 682-691

10. Emilson CG., Gisselsson H, Birkhed D (1999) Recolonisation pattern of mutans streptococci after 
suppression by three different modes of chlorhexidine gel application. Eur J Oral Sci 107, 170-175

11. van Rijkom HM, Truin GJ, van't Hof MA (1996) A meta-analysis of clinical studies on the cariesinhibiting effect of chlorhexidine treatment. J Dent Res 75, 790-795

12. Pratten J, Barnett P, Wilson M (1998) Composition and susceptibility to chlorhexidine of multispecies biofilms of oral bacteria. Appl Environ Microbiol 64, 3515-3519

13. Hepso HU, Bjornland T, Skoglund LA (1988) Sideeffects and patient acceptance of $0.2 \%$ versus $0.1 \%$ chlorhexidine used as post-operative prophylactic mouthwash. Int J Oral Maxillofac Surg 17, 17-20

14. Mackenzie IC, Nuki K, Loe H, Schiott CR (1976) Two years oral use of chlorhexidine in man. V. Effects on stratum corneum of oral mucosa. J Periodontal Res 11, 165-171

15. Takeuchi H, Senpuku H, Matin K, Kaneko N, Yusa $\mathrm{N}$, Yoshikawa E, Ida H, Imai S, Nisizawa T, Abei Y, Kono Y, Ikemi T, Toyoshima Y, Fukushima K, Hanada N (2000) New dental drug delivery system for removing mutans streptococci from the oral cavity: effect on oral microbial flora. Jpn J Infect Dis 53, 211-212

16. Takeuchi H, Fukushima K, Senpuku H, Nomura Y, Kaneko N, Yano A, Morita E, Imai S, Nisizawa T, Kono Y, Ikemi T, Toyoshima Y, Hanada N (2001) Clinical study of mutans streptococci using 3DS and monoclonal antibodies. Jpn J Infect Dis 54, 34-36

17. Caufield PW, Dasanayake AP, Li Y, Pan Y, Hsu J, Hardin JM (2000) Natural history of Streptococcus sanguinis in the oral cavity of infants: evidence for a discrete window of infectivity. Infect Immun 68, 4018-4023

18. Hanada N, Nomura Y, Takeuchi H, Sennpuku H, Ida H, Yosikawa E, Kumagai T (2001) New dental drug delivery system for removing Mutans Streptococci. J Dent Res 80, 567 (abstract)

19. Lewis DW, Ismail AI (1995) Periodic health examination, 1995 update: 2 . Prevention of dental caries. The Canadian task force on the periodic health examination. CMAJ 152, 836-846

20. Curnow MM, Pine CM, Burnside G, Nicholson JA, Chesters RK, Huntington E (2002) A randomized controlled trial of the efficacy of supervised toothbrushing in high-caries-risk children. Caries Res 36, 294-300

21. Axelsson P, Kristoffersson K, Karlsson R, Bratthall D (1987) A 30-month longitudinal study of the effects of some oral hygiene measures on Streptococcus mutans and approximal dental caries. J Dent Res 66, 761-765

22. Binney A, Addy M, Newcombe RG (1993) The plaque removal effects of single rinsings and brushings. J Periodontol 64, 181-185

23. Bellini HT, Arneberg P, von der Fehr FR (1981) Oral hygiene and caries. A review Acta Odontol Scand 39, 257-265

24. Poulsen S, Agerbaek N, Melsen B, Korts D., Glavind L, Rolla G (1976) The effect of professional toothcleansing on gingivitis and dental caries in children after 1 year. Community Dent Oral Epidemiol 4, 195-199

25. Klock B (1984) Long-term effect of intensive caries prophylaxis. Community Dent Oral Epidemiol 12, 69-71

26. Kristoffersson K, Axelsson P, Bratthall D (1984) Effect of a professional tooth cleaning program on interdentally localized Streptococcus mutans. Caries Res $18,385-390$

27. Ullsfoss BN, Ogaard B, Arends J, Ruben J, Rolla G., Afseth J (1994) Effect of a combined chlorhexidine and $\mathrm{NaF}$ mouthrinse: an in vivo human caries model study. Scand J Dent Res 102, 109-112

28. Pienihakkinen K, Soderling E, Ostela I, Leskela I, Tenovuo J (1995) Comparison of the efficacy of $40 \%$ chlorhexidine varnish and $1 \%$ chlorhexidine-fluoride gel in decreasing the level of salivary mutans streptococci. Caries Res 29, 62-67

29. Costerton JW, Stewart PS, Greenberg EP (1999) Bacterial biofilms: a common cause of persistent infections. Science 284, 1318-1322 